\title{
Adaptive developmental plasticity: Compartmentalized responses to environmental cues and to corresponding internal signals provide phenotypic flexibility
}

Ana Rita A Mateus ${ }^{1,2}$, Manuel Marques-Pita ${ }^{1,3+}$, Vicencio Oostra ${ }^{2,4+}$, Elvira Lafuente ${ }^{1}$, Paul M Brakefield ${ }^{2,4}$, Bas J Zwaan ${ }^{5}$ and Patrícia Beldade ${ }^{1,2^{*}}$

\begin{abstract}
Background: The environmental regulation of development can result in the production of distinct phenotypes from the same genotype and provide the means for organisms to cope with environmental heterogeneity. The effect of the environment on developmental outcomes is typically mediated by hormonal signals which convey information about external cues to the developing tissues. While such plasticity is a wide-spread property of development, not all developing tissues are equally plastic. To understand how organisms integrate environmental input into coherent adult phenotypes, we must know how different body parts respond, independently or in concert, to external cues and to the corresponding internal signals.

Results: We quantified the effect of temperature and ecdysone hormone manipulations on post-growth tissue patterning in an experimental model of adaptive developmental plasticity, the butterfly Bicyclus anynana. Following a suite of traits evolving by natural or sexual selection, we found that different groups of cells within the same tissue have sensitivities and patterns of response that are surprisingly distinct for the external environmental cue and for the internal hormonal signal. All but those wing traits presumably involved in mate choice responded to developmental temperature and, of those, all but the wing traits not exposed to predators responded to hormone manipulations. On the other hand, while patterns of significant response to temperature contrasted traits on autonomously-developing wings, significant response to hormone manipulations contrasted neighboring groups of cells with distinct color fates. We also showed that the spatial compartmentalization of these responses cannot be explained by the spatial or temporal compartmentalization of the hormone receptor protein.
\end{abstract}

Conclusions: Our results unravel the integration of different aspects of the adult phenotype into developmental and functional units which both reflect and impact evolutionary change. Importantly, our findings underscore the complexity of the interactions between environment and physiology in shaping the development of different body parts.

Keywords: Bicyclus anynana, Developmental recombination, Ecdysone, Environmental input, Modularity, Phenotypic flexibility, Physiology, Seasonal polyphenism, Thermal plasticity, Trait-specific sensitivities

\footnotetext{
* Correspondence: pbeldade@igc.gulbenkian.pt

${ }^{\dagger}$ Equal contributors

'Instituto Gulbenkian de Ciência, Rua da Quinta Grande 6, P-2780 Oeiras,

Portugal

${ }^{2}$ Institute of Biology, Leiden University, Sylviusweg 72, 2333, BE Leiden, The

Netherlands

Full list of author information is available at the end of the article
}

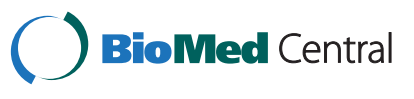

(C) 2014 Mateus et al.; licensee BioMed Central. This is an Open Access article distributed under the terms of the Creative Commons Attribution License (http://creativecommons.org/licenses/by/4.0), which permits unrestricted use, distribution, and reproduction in any medium, provided the original work is properly credited. The Creative Commons Public Domain Dedication waiver (http://creativecommons.org/publicdomain/zero/1.0/) applies to the data made available in this article, unless otherwise stated. 


\section{Background}

In numerous species, the external environment can affect development and lead to the production of distinct phenotypes from the same genotype [1]. This phenomenon is called developmental plasticity. The resulting alternative phenotypes can be as dramatically different as the nutrition-induced differences between workers and queens in social insects (for example, [2-4]) and the seasonal forms of many insects (for example, [5-7]). All organisms have traits that are plastic. However, not all body parts of plastic organisms are equally flexible (for example, [8-10]). The ability of tissue development to both resist and integrate environmental input is crucial for organismal fitness in heterogeneous environments. An important step towards understanding how organisms can adaptively respond to the environment by expressing alternative phenotypes, and organize this response across body parts and traits, is to determine to which degree and by what mechanism body parts are integrated into coordinated modules that correspond to functional, evolutionary and/or developmental units $[11,12]$. This will include understanding how different body parts respond to external environmental cues, as well as to the internal signals that convey information about those cues to the developing tissues.

In insects, ecdysteroid hormones work as internal signals that mediate key developmental transitions, such as molting and metamorphosis, and can also mediate developmental plasticity [7]. The external environment typically affects systemic hormone titers which, in turn, affect developing tissues. So that different traits which respond to the same hormone signal can develop and evolve independently, hormone effects need to be compartmentalized in time and space [7,13]. This type of compartmentalization has been characterized in relation to the environmental regulation, mostly by nutrition, of the growth of different organs during insect larval development $[10,14,15]$. Much less is known about the compartmentalization of hormone effects for different groups of cells within the same tissue, and during postgrowth tissue patterning. We investigate this process here for an evolutionary ecology model of developmental plasticity.

The butterfly Bicyclus anynana has become a textbook example of adaptive developmental plasticity [1,16-19]. Its study combines knowledge about the ecological and evolutionary significance of plasticity with the analysis of its genetic and physiological underpinnings $[1,20]$. In natural populations, butterflies developing in the dry versus the wet season have cryptic versus conspicuous ventral wing patterns, each associated with different seasonal strategies to avoid predation [1]. The wing phenotypes encompass a whole suite of pattern elements which differ between the seasons. In the laboratory, the development of wet- versus dry-like phenotypes can be induced by the temperature experienced during pre-adult stages [1]: warmer temperatures induce wet-like wing patterns, while cooler temperatures induce dry-like phenotypes. Previous studies showed differences between warm- versus cool-reared pupae in the dynamics of ecdysone levels [21] (Figure 1A) and established these as a cause for changes in wing pattern [21]. Various studies of B. anynana wing pattern plasticity characterized the effects of the temperature and/or ecdysteroid levels on a few indicative pattern traits [22-25]. Limiting these analyses to only a few traits has precluded an assessment of how the effects of external and internal signals are compartmentalized in the developing wings. A systematic analysis of both types of cues on multiple aspects of wing patterns is lacking.

To characterize the effects of external cues and internal signals on tissue patterning, we manipulated temperature during pre-adult development and manipulated the levels of active ecdysone in the pupal hemolymph (Figure 1). We then compared the suite of adult wing traits that constitute the seasonal wing phenotype. The traits we chose (Figure 2) reflect increasing levels of spatial resolution in the analysis of the compartmentalization of plasticity. They allow comparisons between: 1) different wings derived from autonomously-developing imaginal discs (fore- and hindwing); 2) different surfaces of the same wing that correspond to distinct cell sheets (dorsal and ventral surfaces) and evolve under different selection regimes [26]; 3) different types of pattern elements (eyespots and band) displaying weak genetic correlations between them; 4) different repeats of the same type of pattern element (anterior and posterior eyespots on the same wing surface) with stronger correlations between them [19,27]; and 5) different rings of the same eyespot (central white focus, middle black disc, and external golden ring) that correspond to groups of neighboring cells responding to a morphogen signal originated at each presumptive eyespot center [19,28-31]. Our data on this extensive set of traits allow us to investigate the coordination of responses to external cues and internal signals across groups of wing epidermal cells and the mechanism for the spatial compartmentalization of the sensitivities to those signals. We discuss our results in terms of whether tighter or looser integration between traits might be adaptive and/or might represent (constrained) properties of the development in response to environmental variation.

\section{Results and discussion}

Our results show that different groups of cells on the developing wing epidermis, which correspond to different aspects of the color pattern on adult female wings, have characteristic sensitivities to changes in temperature during pre-adult development (Figure 3), as well as to changes in ecdysone levels during the pupal stage (Figure 4). We could identify not only which traits are, and are not, 

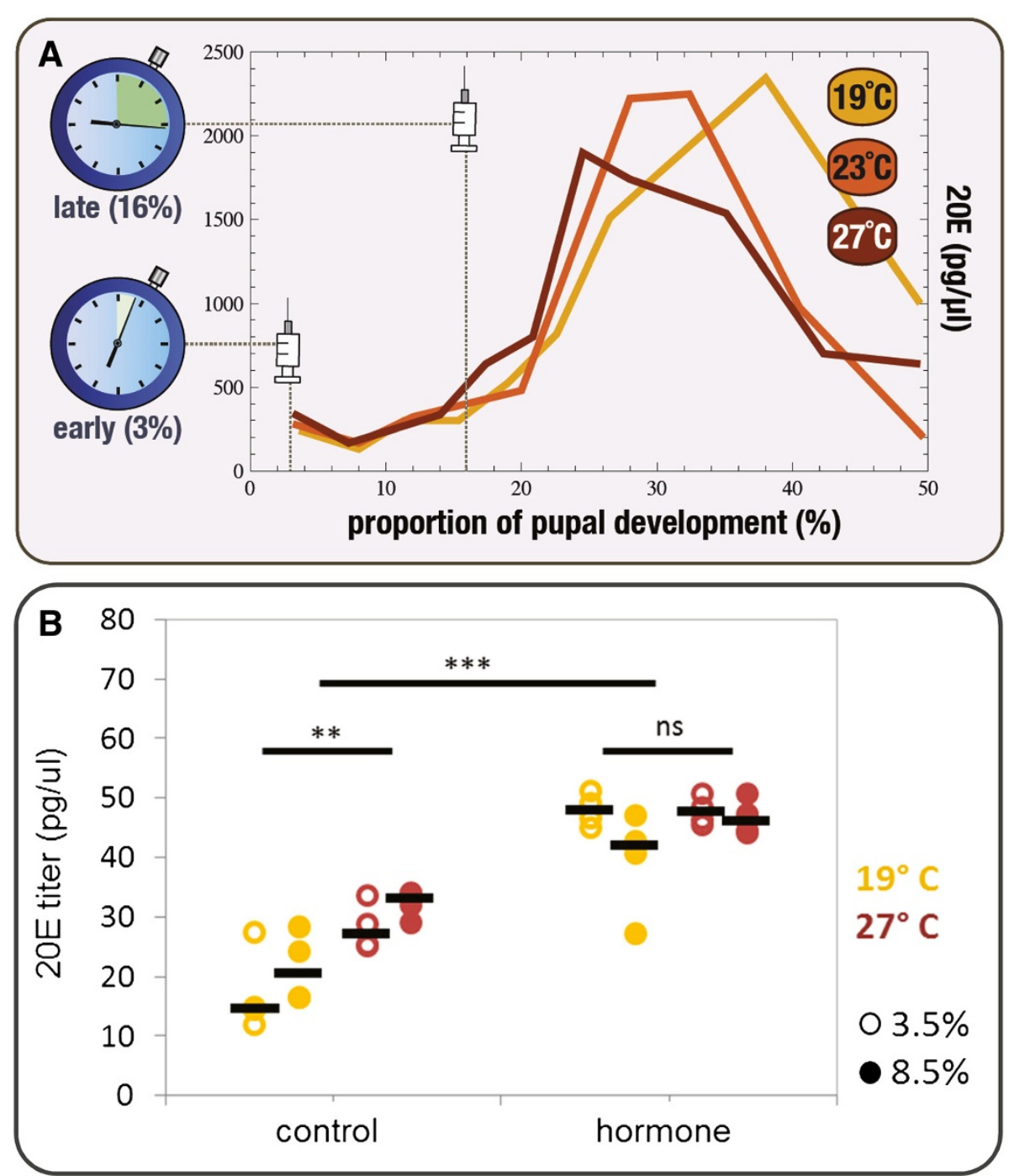

Figure 1 Dynamics and manipulation of internal levels of ecdysone. (A) Experimental design for hormone manipulations. Hydroxyecdysone (20E) injections were done on female pupae reared at $19^{\circ} \mathrm{C}, 23^{\circ} \mathrm{C}$ or $27^{\circ} \mathrm{C}$ at two developmental stages corresponding to different phases of the natural 20E dynamics (compare with [21]): 'early', before ecdysone concentration starts to increase (at 3\% of the total time it takes to complete pupal development at each of the temperatures), and 'late', corresponding to the ascending phase of the ecdysone level (at 16\% of the total pupal development time). (B) Effect of early hormone injections on hormone titers. Internal levels of 20E at 3.5\% and $8.5 \%$ of total pupal development time after early injection of hormone and control solutions at $19^{\circ} \mathrm{C}$ and $27^{\circ} \mathrm{C}$. The bar represents the median value of four individuals per treatment, temperature, and time point (see Material and methods). We tested for the effect of temperature and injection treatment on the levels of 20E at two time points using the model 20E time point + temperature * injection, for which the residuals showed no significant departure from normality (Shapiro-Wilk W test: $W=0.950, P=0.146)$ or from homogeneity of variances (Fligner-Killeen test: Median Chi Square $=1.176, d f=1, P=0.185)$. The analysis of variance revealed a statistically significant effect of temperature $\left(F_{(1,32)}=13.848, P=0.0009\right)$ and injection $\left(F_{(1,32)}=114.501, P=3.25 \mathrm{e}-11\right)$, but not of time point $\left(F_{(1,32)}=0.026, P=0.874\right)$ or temperature*injection $\left(F_{(1,32)}=3.670, P=0.066\right)$. Results of the post-hoc pairwise comparisons using the Tukey honest significance test are indicated in the figure: $n$ f for $P>0.01,{ }^{* *}$ for $P<0.01,{ }^{* * *}$ for $P<0.001$ [see Additional file 4 for more on this analysis].

responsive to manipulations of the external cue and internal signal, but also identify groups of sensitive traits that display distinct patterns of coordinated responses (Figure 5). Finally, we show that the spatial compartmentalization of hormone sensitivities is not due to the spatial or temporal compartmentalization of the hormone receptor protein (Figure 6).

\section{Response of wing traits to developmental temperature}

To assess how different groups of cells on the developing wings respond to external environmental cues, we measured wing patterns of butterflies reared at three temperatures, representing typical wet- and dry-inducing extremes $\left(27^{\circ} \mathrm{C}\right.$ and $19^{\circ} \mathrm{C}$, respectively) and an intermediate temperature $\left(23^{\circ} \mathrm{C}\right)$. We then compared phenotypes between 

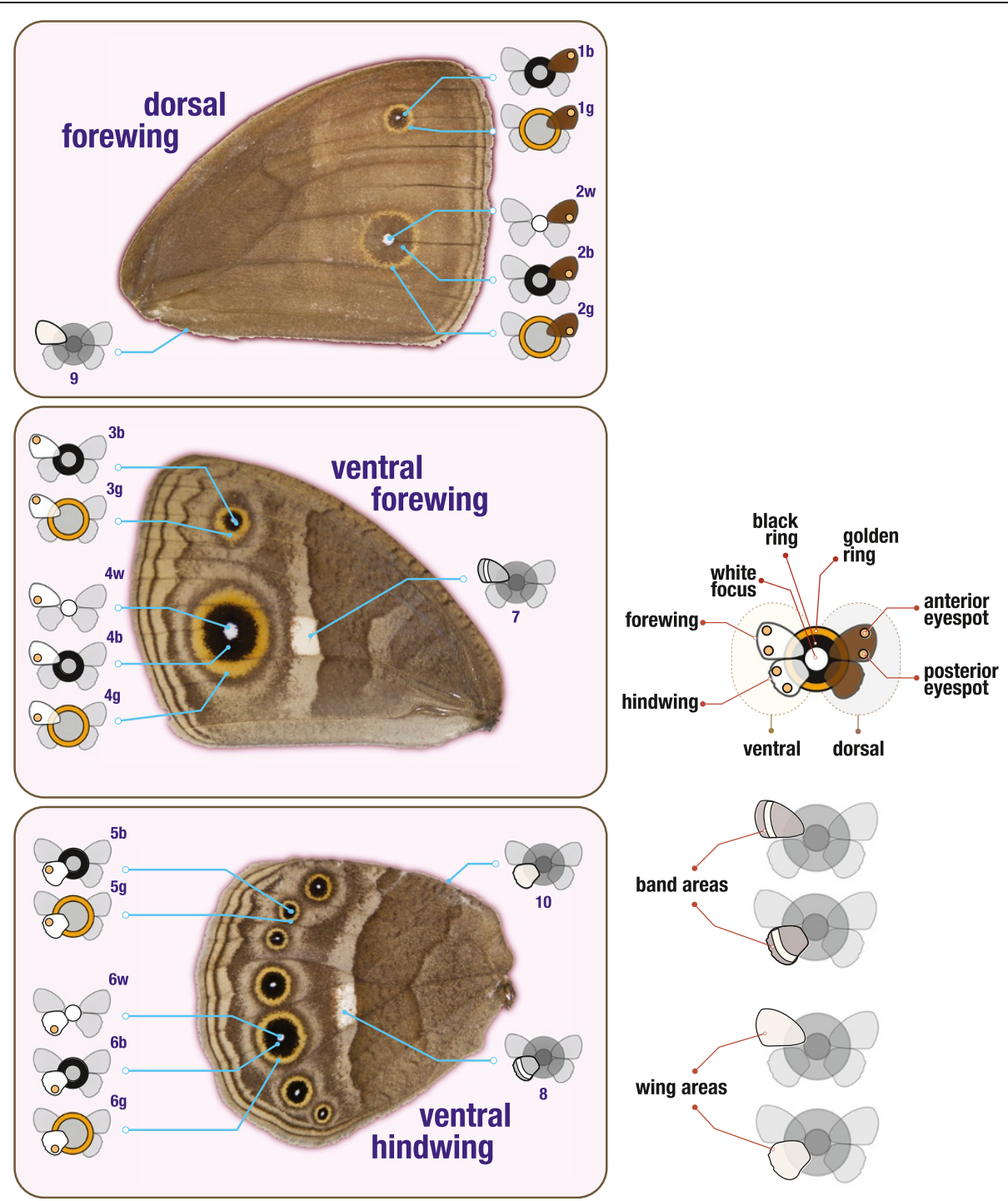

Figure 2 Wing traits measured in adult females. The photos represent the typical phenotype of female Bicyclus anynana reared at $27^{\circ} \mathrm{C}$. Note that the dorsal surface of the hindwing does not always have color patterns beyond occasional extra eyespots or just their white pupils which are generally too small for accurate size measurements. For each individual, we obtained 19 wing measurements corresponding to four categories of traits: dorsal eyespots, ventral eyespots, ventral band and wing areas. Note that each eyespot corresponds to a different trait number and we use different letter codes to refer to the corresponding white centers ( $w$ ), black discs (b) and golden rings ( $\mathrm{g}$ ). The diagram on the right panel displays the symbols used to refer to each of the traits in the other figures. On each wing surface (ventral represented in white and dorsal in brown), we measured two eyespots (one more anterior represented by a circle on the top and one more posterior by a circle on the bottom). The color of the circles at the center of the image corresponds to each of the three color rings that make up each eyespot.

temperatures. Figure 3 shows the thermal reaction norms for the 19 target traits in adult females. For the first time, this involved considering separately and simultaneously the distinct color rings (white, black, and gold) of multiple eyespots on different parts (anterior and posterior) of the same wing surface and on different wing surfaces (ventral and dorsal) (Figure 2).

This extensive analysis of wing pattern traits revealed that, in contrast to what had been described, some aspects of the dorsal wing pattern are plastic in relation to developmental temperature (Figure 3A). Previous studies of plasticity on dorsal forewing color pattern had investigated the most posterior eyespot (our trait 2) and found it to be largely non-plastic across seasonal environments $[22,25]$. Our results confirm this but, by also analyzing other pattern elements on the same wing surface, show that the lack of temperature-sensitivity is not a property of the whole dorsal wing surface. The more anterior eyespot on the dorsal forewing (trait 1) did increase significantly with temperature (Figure 3A). As expected from 

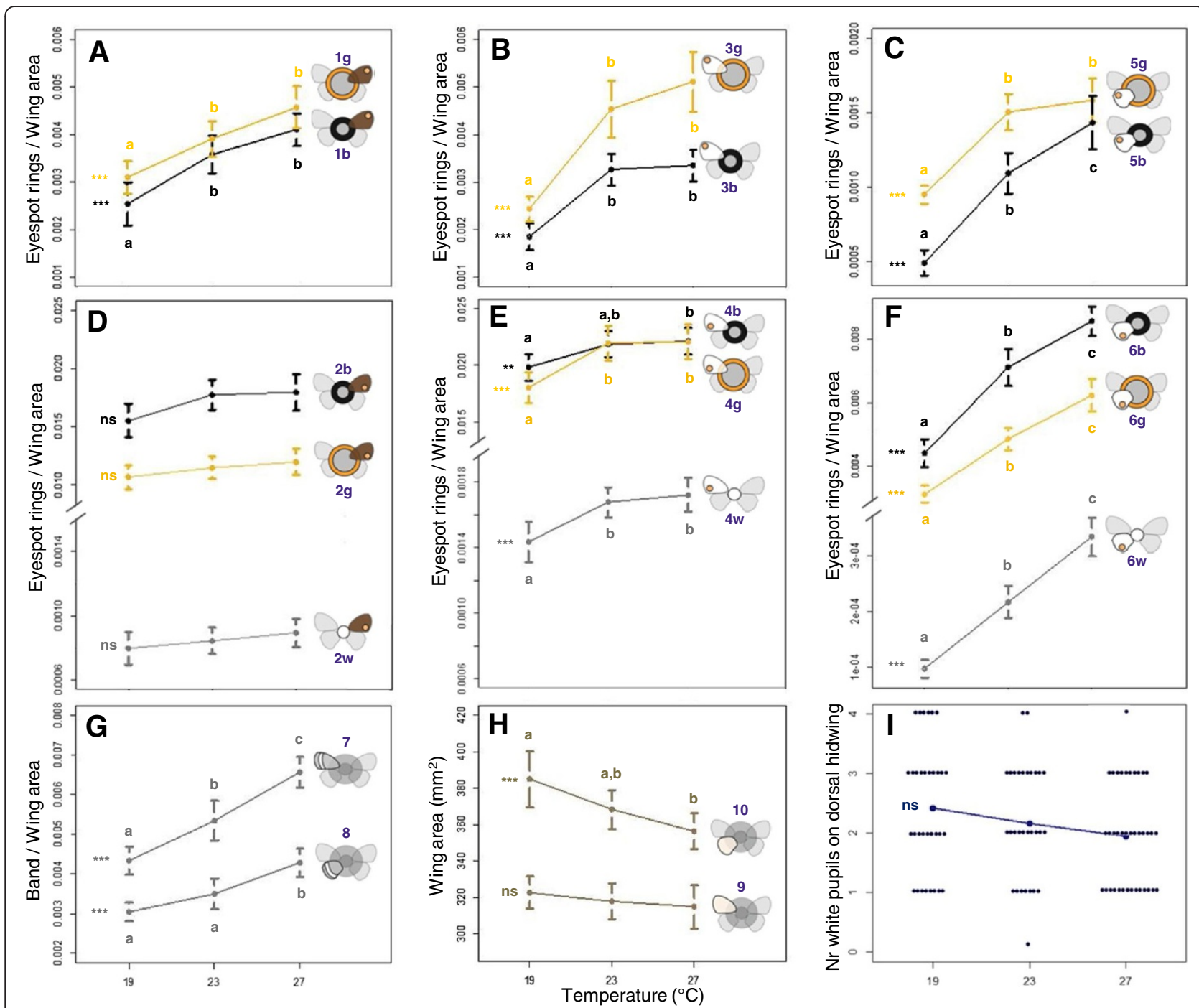

Figure 3 Effect of temperature experienced during development on wing traits. For each trait, we plot the mean value as a function of temperature and use bars to represent the standard deviation for 24 to 38 measurements per temperature. These representations, called reaction norms, are the standard way of displaying plasticity. Trait icons, compare with Figure 2, are given on the right of the respective reaction norm line: (A-B) dorsal eyespots, (C-F) ventral eyespots on forewing and hindwing, $\mathbf{( G )}$ ventral bands and $\mathbf{( H )}$ wing areas. We tested for the effect of temperature on wing pattern trait using the model trait temperature + wing (where the area of the corresponding wing is a covariate) and on wing area using wing temperature (see Material and methods). Trait values were used untransformed or log 10 transformed to meet the ShapiroWilk normality test (alpha $=0.05$ ). Statistical significance for effects of temperature on wing traits (see Material and methods) is indicated to the left of each reaction norm: ns (non-significant), $P>0.01,{ }^{* *} P<0.01,{ }^{* * *} P<0.001$. When ANCOVA/ANOVA showed significant effects of temperature on trait value, we compared across temperatures. For each reaction norm, different letters indicate pairwise comparisons that revealed statistically significant differences (Ismeans $P<0.01$ ) (see Additional file 1 for more details on these statistical analyses). For the number of white pupils ( $n=30$ to 38 individuals, Additional file 1) on the dorsal surface of the hindwing in panel (I), we found no significant effect of temperature using the model pupil $\mathrm{nr} \sim$ temperature with a quasi-Poisson distribution (Deviance $=1.894, d f=2, P=0.1172$ ). ANCOVA, analysis of covariance; ANOVA, analysis of variance.

previous studies, wing pattern components on the ventral surface of the wings showed clear thermal plasticity (Figure 3B, C, E, F, G; see Additional file 1).

\section{Only the wing pattern element implicated in mate choice does not respond to temperature}

Previous work largely focused on ventral wing patterns because this is the surface exposed to predators in butterflies at rest and, thus, the surface under predator-driven natural selection for plasticity [20]. Seasonal variation in ventral wing patterns is associated with seasonal variation in the resting background and to alternative strategies for butterflies to avoid predation. In the cooler dry season, duller brown wing patterns with no striking color elements are cryptic in relation to the resting background of dry brown leaves. In the warmer wet season, more 


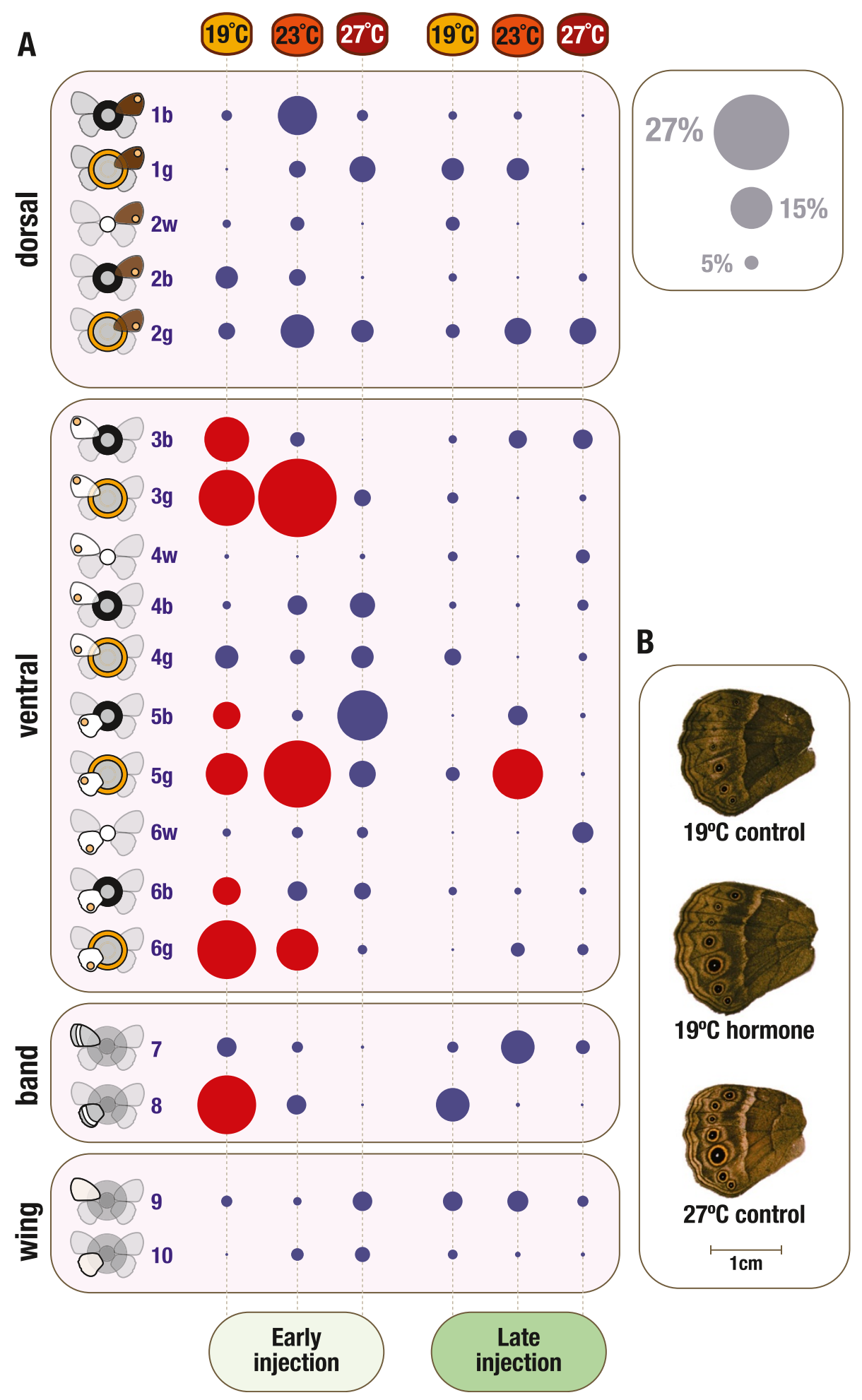

Figure 4 (See legend on next page.) 
(See figure on previous page.)

Figure 4 Effect of pupal hormone manipulations on different wing traits. (A) For each trait, temperature and time point combination, the circles represent the magnitude (circle size; scale on top right corner) and statistical significance (circle color, with red for significant differences; compare with permutation test explained in the Material and methods) of the difference between hormone- versus control-injected individuals (details in Additional file 2). As for Figures 2 and 3, the traits are organized per type: dorsal eyespot traits, ventral eyespot traits, ventral bands and wing areas. The final number of measurements for each trait in each experimental group can be found in Additional file 2. The difference between control and hormone treatments was tested using a series of core and confirmatory statistical tests, all giving largely the same results (details in Materials and methods and Additional file 2). (B) Photos of the ventral surface of adult hindwings representing the phenotypes of different temperature and injection treatments: control-injected individual at $27^{\circ} \mathrm{C}$, hormone-injected individual at $19^{\circ} \mathrm{C}$ and control-injected individual at $19^{\circ} \mathrm{C}$. Scale bar corresponds to $1 \mathrm{~cm}$. All images are from butterflies injected as pupae at 3\% of their development time. These wings illustrate how early hormone manipulations at lower temperature increase the area of different color pattern components, bringing the phenotypes closer to those of individuals reared at higher temperature.

conspicuous color elements along wing margins can function as targets for predator attacks away from the more fragile body [1,32].

The dorsal patterns, on the other hand, are typically not exposed in the butterfly at rest and presumably not under selection by predators. Instead, those patterns are exposed during courtship and thought to evolve under sexual selection $[25,26,33]$. In particular, some of the UVreflecting white pupils of dorsal eyespots have been shown to influence mate choice [25,34]. In our study of female butterflies, the only eyespot that showed no significant response to temperature (Figure 3D; trait 2) was the one that is sexually selected in males [25]. The white center of this eyespot had been found to be plastic in males; being larger and more UV-reflecting in wet season courting individuals [25]. Even though it has been proposed that dry season females do courtship [25], in a case of seasonallyplastic sexual selection, we found that the corresponding trait is not plastic in females (Figure 3D; trait $2 \mathrm{w}$ ). Instead, a recent study proposed that male choice among potential dry-season mating partners depends on the number of white pupils found on the dorsal surface of the female hindwing [34]. The number of such pupils was shown to vary between females reared at $17^{\circ} \mathrm{C}$ versus $27^{\circ} \mathrm{C}$ [34]. In our study, we found that the mean (but not the median) number of white pupils on the ventral surface of the hindwing of non-injected females decreases with increasing temperature, but not significantly so (Figure 3I).

\section{Response of wing traits to hormone manipulations}

To examine how different groups of cells on the wings respond to changes in hormone levels, we measured the effect of hormone manipulations during the early pupal stage when the signaling from eyespot organizers and the response of the surrounding cells to the ring-determining morphogen are known to take place [19]. We manipulated the levels of active ecdysone in the hemolymph by injecting female pupae with 20-hydroxyecdysone (20E) [22-24] at two developmental time points (Figure 1). For each temperature and injection time point, we then compared adult wings between control-injected and hormoneinjected individuals. Figure 4 shows the magnitude and statistical significance of the difference between control and hormone treatments for each of the target traits, injection time points, and rearing temperatures [see also Additional file 2].

Only traits that responded to changes in temperature during development responded to changes in hormone titers during early pupal life. That is, all traits for which differences between control-injected and hormone-injected individuals were significant (that is, any red circles in Figure 4) are traits for which the differences between temperatures for non-injected individuals were also significant (that is, reaction norms marked with stars in Figure 3). However, not all wing pattern traits that responded to temperature were affected by the hormone treatment. We found no significant effect of hormone manipulations for any of the traits in the dorsal wing surface (Figure 4A). In contrast, many traits on the temperatureplastic ventral wing surfaces significantly increased in area in response to hormone injections. In some cases, lack of effect of our hormone injections on temperatureresponsive traits can be explained by the fact that trait determination occurred before the hormone treatment. This is the case for the white eyespot centers (traits $4 \mathrm{w}, 6 \mathrm{w}$ in Figure 2) and for hindwing area (trait 10). The establishment of the eyespot organizing centers [35] and most of wing growth [36] are known to take place during larval life, before our hormonal injections were done. However, for other non-responsive traits, notably eyespot color rings, that is not the case (see below).

\section{Only pattern elements on the wing surface exposed to predators respond to changes in pupal ecdysone levels}

For all dorsal (traits 1 and 2) and some ventral thermallyresponsive color pattern elements (traits 4 and 7) that did not respond to hormone treatment, it seems unlikely that our treatment missed the relevant windows of trait determination. Certainly for eyespot rings, we know that it is during early pupal development that signaling from eyespot centers establishes concentric rings of cells fated to produce different color pigments [30,31]. The lack of response of those traits to our hormone manipulations could be due to lower sensitivities to hormone titers and 


\section{A. Response to environmental input (temperature)}

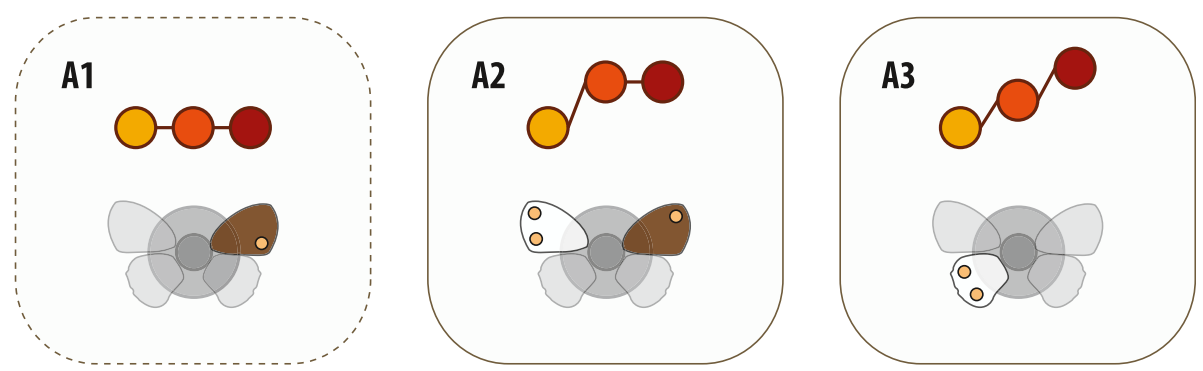

\section{B. Response to systemic signal (hormone manipulation)}

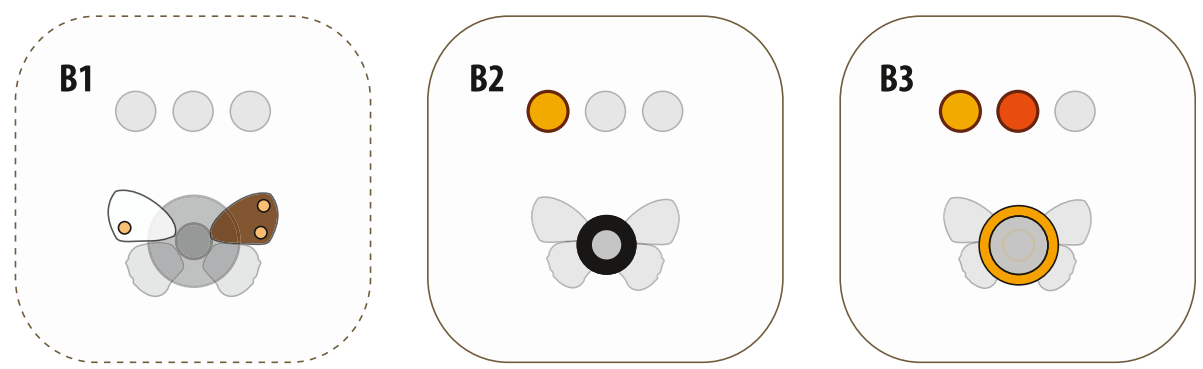

\section{Selection on adult eyespot traits}

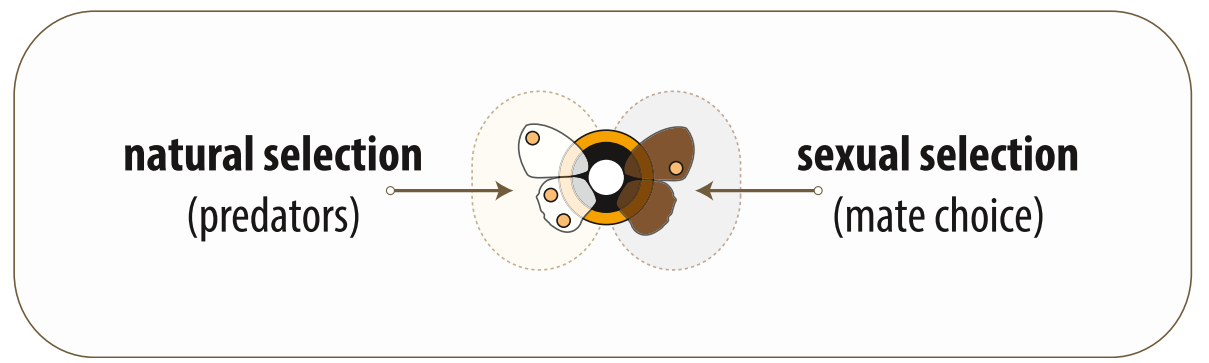

Figure 5 Patterns of coordinated response to external and internal signals. Each box includes eyespot traits that responded in a similar manner to differences in developmental temperature (A) and to hormone injections (B). Boxes in dashed lines represent traits (symbols, compare with Figure 2) that do not respond to temperature (A1) or to hormone injections (B1). The other boxes represent distinct patterns of response to temperature (A2-A3) or to ecdysone (B2-B3) [see details in Additional file 5]. The three circles at the top of each box represent each of the three experimental temperatures: from right to left, $19^{\circ} \mathrm{C}, 23^{\circ} \mathrm{C}$ and $27^{\circ} \mathrm{C}$. In panel (A), lines between those circles illustrate the shapes of the corresponding thermal reaction norms (compare with Figure 3): flat for $\mathrm{A} 1,19^{\circ} \mathrm{C}<23^{\circ} \mathrm{C}$ approximately $27^{\circ} \mathrm{C}$ for $\mathrm{A} 2$, and $19^{\circ} \mathrm{C}<23^{\circ} \mathrm{C}<27^{\circ} \mathrm{C}$ for $\mathrm{A} 3$. In panel (B), the circles not in gray represent temperatures for which phenotypes were significantly different between control-and hormoneinjected individuals (compare with Figure 4): no effect of hormone manipulations for whichever temperature in $\mathrm{B} 1$, effect only for $19^{\circ} \mathrm{C}$ in $\mathrm{B} 2$ and effect both at $19^{\circ} \mathrm{C}$ and $23^{\circ} \mathrm{C}$ in $\mathrm{B} 3$. The only traits that do not respond to temperature (A1) correspond to the eyespot shown to be under sexual selection, while those that do not respond to hormone manipulations (B1) are those not exposed to predators in resting butterflies (C). The patterns of response to temperature contrast fore- and hindwing while those for hormone manipulations contrast black and golden color rings. A detailed scheme of the patterns of response showing all traits can be found in Additional file 5.

due to them requiring hormone concentrations higher than those we produced artificially. This, too, at least alone, seems unlikely because our post-injection hormone levels at $19^{\circ} \mathrm{C}$ surpassed the control levels at $27^{\circ} \mathrm{C}$, a temperature difference for which the traits did change (see below and Figure 1B). The lack of response to hormonal manipulations suggests that thermal plasticity for these traits is not mediated (exclusively) by ecdysone.
It is curious to note that the color traits established in early pupae which we found to be thermally-sensitive but ecdysone-resistant are presumably under no, or weaker, selection by predators. As discussed before, this is the case for color patterns on the dorsal surface of the wing which is not exposed in the butterflies resting against the seasonally color-variable background foliage. Also, unlike other ventral pattern elements, the wing region 
containing the hormone-unresponsive traits 4 and 7 is typically covered by the hindwing in the resting butterfly. Therefore, these traits too are presumably less exposed to the predators that drive selection for seasonally plastic ventral wing patterns. A weaker selection pressure by natural enemies could explain why these particular traits evolved different levels of plasticity.

\section{Levels and time windows of sensitivity to hormone manipulations}

All traits that responded to hormone injection treatment (Figure 4) were larger in hormone-treated relative to control-treated butterflies. The hormone-induced increase in size is consistent with the temperature plasticity: development at warmer temperatures, associated with an earlier increase in natural 20E titers [21-24] (see Figure 1A), leads to the production of more conspicuous wing patterns with larger areas of non-background color (Figure 3). By artificially increasing hormone levels at the lower temperatures, we induced the production of the same type of phenotypic effect that higher temperatures have on wing patterns (Figure 4B; see also Additional file 3 ). The fact that the artificial increase in hormone levels phenocopied the temperature effect confirms a role for ecdysteroids at this early-pupal developmental stage in mediating thermal plasticity in wing patterns.

Strikingly, we detected the strongest responses to hormone manipulations for injections done at the early developmental time point, when the natural levels of pupal ecdysone are very low and differences between temperatures were previously undetectable [21], and not for injections at the later time point when hormone titer differences between temperatures are clear (Figure 1). This suggests a window of sensitivity to the hormone between our two injection time points, that is, between $3 \%$ and $16 \%$ of pupal life. For only one of the target traits (trait $5 \mathrm{~g}$ ), did we see an effect of later hormone manipulation. This indicates some level of heterochrony in the development of this trait, which appears to have a later window of sensitivity to the hormone. Heterochrony, differences in the developmental times and/or rates, is an important contributor to phenotypic diversification, including for butterfly wing patterns $[37,38]$. We have shown previously that hormone manipulations at later time points do affect a number of life-history traits [39].

We did not observe significant effects of hormone manipulations at higher temperatures (Figure 4), even if our manipulations did significantly change hormone titers. We measured 20E concentration in the hemolymph of pupae at $3.5 \%$ and $8.5 \%$ of pupal development time for the two extreme experimental temperatures after early injection of hormone and of control solutions (Figure 1B). Hormone levels are significantly higher for hormoneinjected versus control-injected pupae at both rearing temperatures [see Additional file 4]. Control pupae show higher $20 \mathrm{E}$ levels when reared at $27^{\circ} \mathrm{C}$ relative to $19^{\circ} \mathrm{C}$, consistent with the relatively faster increase in natural hormone titers that occurs at higher temperatures (Figure 1A). After hormone injection we can no longer detect differences in internal levels between temperatures (Figure 1B).

\section{Differences in trait associations in response to external and internal cues}

Focusing on the eyespot traits that are plastic in relation to temperature and/or to hormone titers, we can identify different categories of response [see Additional file 5 summarized in Figure 5]. The principal component analyses [see Additional file 6], a standard approach for analyses of multidimensional datasets such as ours, identified traits with similar and contrasted responses but not with the same resolution as our analyses of individual traits (compare Figures 3 and 4).

The groups identified based on the response to temperature largely contrast eyespots on the forewing versus hindwing (Figures 3 and 5A). All forewing eyespot traits are significantly smaller at $19^{\circ} \mathrm{C}$ and do not differ between $23^{\circ} \mathrm{C}$ and $27^{\circ} \mathrm{C}$, while all hindwing eyespot traits significantly increase in size with temperature. In summary, for the effects of temperature on wing patterning, we observed looser integration across autonomously-developing wings and tighter coordination of traits on the same wing. The single hindwing trait (trait $5 \mathrm{~g}$ ) that responds to temperature in the same manner as all forewing traits (Figure 3 and Additional file 5: Figure S2A) is also the only trait significantly affected by late hormone manipulations (Figure 4). It is unclear what, developmentally or ecologically, might be the uniqueness of this trait.

For the traits that we found to be sensitive to early manipulations of pupal hormone levels, we found a different pattern of coordinated responses. Because 1) color rings of each eyespot are specified by the same morphogen gradient established from each eyespot's center $[19,40], 2)$ each eyespot center produces morphogen independently of other eyespots [30], and 3) eyespot centers have been shown to have higher levels of ecdysone receptor protein [41], we had hypothesized that all rings of a single eyespot would respond to hormone manipulations in concert and relatively independently from those of other eyespots $[19,28]$. However, rings of the same color, and not rings of the same eyespot, responded in a similar manner (Figures 4 and 5B). All plastic black rings showed hormone-related changes only at $19^{\circ} \mathrm{C}$ while all golden rings showed hormone-related changes both at $19^{\circ} \mathrm{C}$ and $23^{\circ} \mathrm{C}$ (Figure 4). Among the golden rings, we can further distinguish between those from the anterior versus the posterior-half of the wings. They differ in relation to how much hormone-related change we saw at $19^{\circ} \mathrm{C}$ versus $23^{\circ} \mathrm{C}$ (Figure 4, Additional file 5: Figure S2B). 


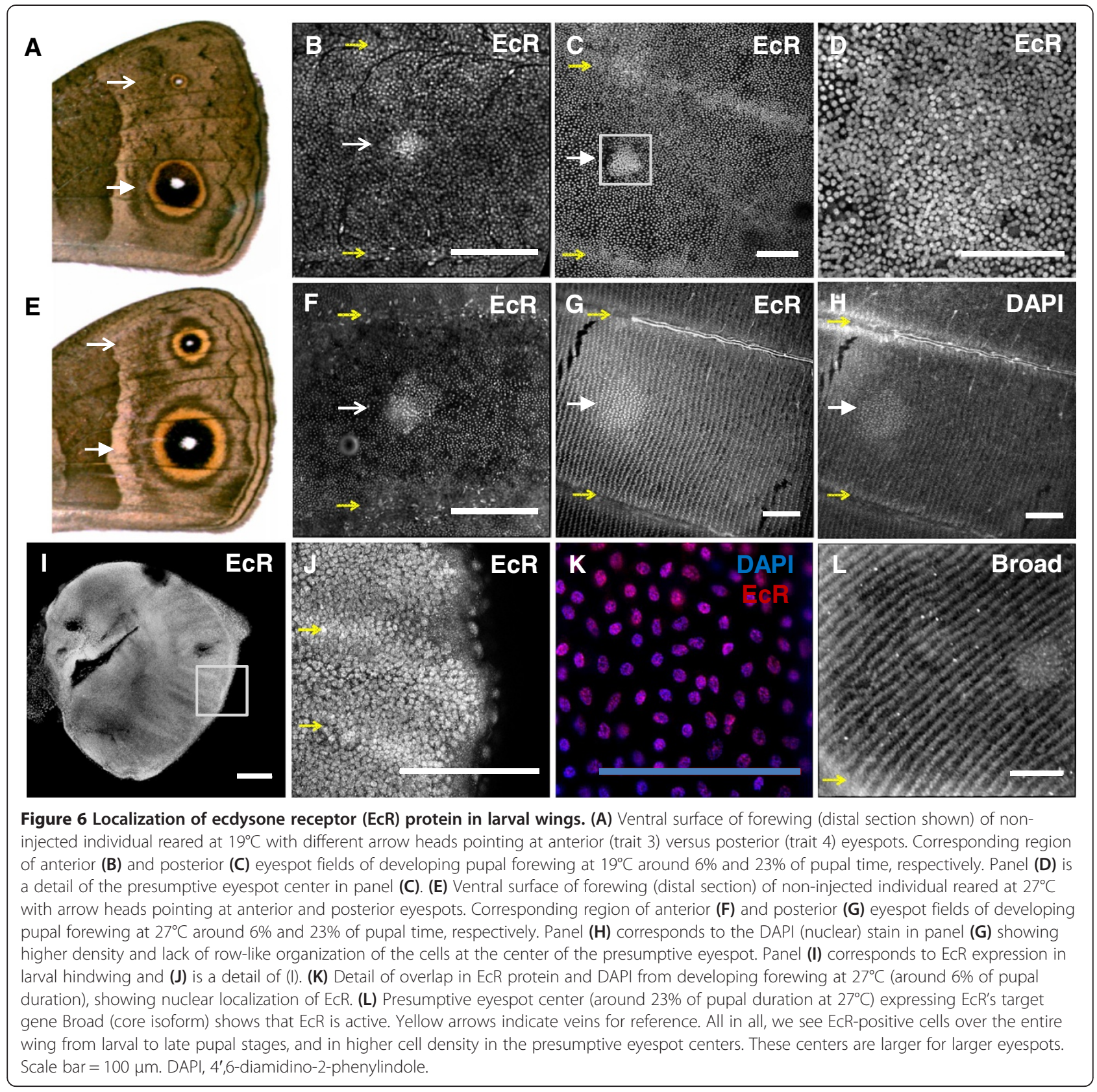

This is consistent with studies showing coupling of anterior (and of posterior) portions across wing surfaces [27] and uncoupling of anterior versus posterior eyespots within the same wing surface $[28,42]$.

\section{Compartmentalization of hormone effects is not explained by hormone receptor localization}

As a mechanism for local sensitivities to systemic levels of $20 \mathrm{E}$, we hypothesized that groups of cells that responded differently to $20 \mathrm{E}$ manipulations would differ in expression of ecdysone receptor (EcR). To test this hypothesis, we investigated the localization of EcR protein in wings from pupae reared at different temperatures using an antibody against B. anynana's EcR [43]. We found EcR in cells on the entire pupal wing epidermis at all temperatures and throughout the whole early pupal life, extending well after the $16 \%$ of developmental pupal time used as our last injection time point (Figure 6). The density of EcR-positive cells was higher in circular regions corresponding to the eyespot organizing centers [41]. These regions were smaller for pupae reared at $19^{\circ} \mathrm{C}$ relative to $27^{\circ} \mathrm{C}$ (Figure $6 \mathrm{~B}, \mathrm{C}$ versus $6 \mathrm{~F}, \mathrm{G}$; [44]), and for smaller versus larger eyespots (Figure 6B, F versus 6C, G). 
Surprisingly, however, this pattern of EcR expression was detected both for the highly plastic ventral and the hormone-unresponsive dorsal eyespots. This shows that the non-responsiveness of the dorsal color traits to hormone manipulations cannot be due to the corresponding cells not having the receptor for the systemic signal, as had been previously proposed [22]. Our data also did not reveal visible differences in EcR levels between the regions of the presumptive black versus golden eyespot rings (Figure 6B-D and 6F-G) that showed different sensitivities to the hormone injections (Figure 5). This indicates that differences in the way they respond to hormone manipulations (Figure 5B) must be determined either upstream of the binding of $20 \mathrm{E}$ to its receptor in the cell nucleus (for example, cell permeability to hormone) or downstream of that (for example, factors interacting with the activated EcR (compare with [14]).

\section{Conclusions}

Environmental cues can have systemic effects but also localized effects in developing organisms. These are typically mediated by hormone signals in the circulating hemolymph which carry the information about the external environment to the developing tissues. However, not all organs and groups of cells within organs have equal sensitivities to the external cues and internal signals. The compartmentalization of these effects reflects what has been called phenotypic integration to imply tight connections between traits or phenotypic independence to refer to connections that are readily uncoupled (discussed in [13]). The present study identified such differing modes of connections for different aspects of butterfly wing patterns in relation to external temperature and to internal levels of ecdysone. With our systematic analysis of multiple traits in different temperature and hormone contexts (Figures 1 and 2), we have: 1) identified which traits are, and which are not, responsive to temperature during development (Figure 3), and to changes in ecdysone levels in early pupal life (Figure 4); 2) identified which of the sensitive traits respond in concert to each of the cues, and shown that these groupings are not the same for both types of cues (Figure 5); and, finally, 3) revealed that the mechanism for spatial compartmentalization of the responses does not reflect the spatial or temporal compartmentalization of the receptor for the internal signal (Figure 6).

\section{Overview of the effects of developmental temperature and ecdysone manipulations on plastic wing patterning} We found unexpected differences between sensitivity to temperature and to hormone, both in terms of traits that are responsive versus those that are unresponsive, and also in terms of the traits that respond in a coordinated manner (Figure 5). In relation to the effects of external temperature on wing patterning, we showed that all color traits increase in size with increasing temperature (Figure 3) with the exception of the rings of a single eyespot (Figure 3D; Figure 5A1 and C) previously shown to be under sexual selection in males [25]. Among the temperature-sensitive eyespot traits, we found that all color elements on the forewing respond in the same fashion and differently from all but one color element on the hindwing [see Additional file 5: Figure S2A, summarized in Figure 5A]. The contrast between fore- and hindwing is consistent with the hypothesis that traits on autonomously-developing organs are more loosely integrated than traits on the same organ.

In relation to the effect of increasing hormone levels in early pupal life, we showed that only ventral color patterns, known to be associated with seasonally-plastic strategies for avoiding predators, responded (Figure 4; Figure $5 \mathrm{~B}$ and $\mathrm{C}$ ). Among the hormone-responsive eyespot traits, we found that rings of the same color respond in concert and in a pattern distinct from rings of another color [see Additional file 5: Figure S2B, summarized in Figure 5B]. This contrast is not consistent with the hypothesis that all rings of the same eyespot show similar sensitivity to hormone levels because they are all specified by a morphogen gradient originating from the eyespot center expressing hormone receptor [41]. We further show that the spatial compartmentalization of hormone effects is not due to the spatial compartmentalization of the levels of hormone receptor protein (Figure 6), as had been suggested [22]. Overall, our results point to complex interactions between the environmental cues that induce developmental plasticity and the internal signals that carry information about those cues to the developing tissues.

\section{Sensitivities to external cues and internal signals are shaped by and impact phenotypic evolution}

The coordinated trait sensitivities are properties of development that may have been favored by selection; for example, because it is important for fitness that traits change in concert. However, they may also be properties of development that are selectively neutral (that is, it is irrelevant whether or not traits develop in concerted fashion) or even evolutionarily constrained (that is, it could be advantageous for traits to change independently but the way they develop makes that difficult [45]). The integration between traits can be a factor constraining future responses to selection if integrated traits are selected to change in opposite ways (evolutionary constraint hypothesis $[13,46])$. On the other hand, having traits responding independently to systemic hormone or external input can allow more rapid evolution of new arrangements of traits (evolutionary potential hypothesis [46]). It has been proposed that trait 'reorganization' 
produced by exposure to novel environmental conditions can lead to the production of new phenotypic variants and differences between species, through a process that has been called developmental recombination [47].

To understand fully this type of phenomenon it will be necessary to expand on studies such as ours. It is fundamental to combine the analysis of how different traits are integrated in their response to internal and external cues with an analysis of the mechanisms of differences in response to those cues and the ecological implications of changes in individual traits. In nature, the integration of all levels of information is further complicated by the fact that the developmental environment is more complex than one single changing cue, the phenotype is more than one particular trait, and the selective environment presents more than one ecological challenge.

\section{Material and methods}

\section{Experimental animals}

We used a large outbred laboratory colony of Bicyclus anynana butterflies [20]. Hundreds of eggs collected from this stock were distributed over three climate-controlled rooms (70\% relative humidity, 12:12 hour light/dark cycle) differing in ambient temperature $\left( \pm 0.5^{\circ} \mathrm{C}\right)$. We chose temperatures that simulate the conditions of the natural dry $\left(19^{\circ} \mathrm{C}\right)$ and wet $\left(27^{\circ} \mathrm{C}\right)$ seasons, and an intermediate temperature $\left(23^{\circ} \mathrm{C}\right)$. Larvae were fed ad libitum with young maize plants. Pre-pupae were collected daily and pupation times determined ( \pm 15 minutes) by timelapse digital photography (Canon EOD 100 camera, GT time-lapse remote control). Female pupae from each temperature were split into three experimental groups: non-injected, injected with control solution, and injected with hormone solution (see below). We started with 28 to 70 per temperature per treatment but final sample sizes were smaller for some groups (for example, due to mortality associated with early hormone injections; see below). For non-injected butterflies, we obtained 33 females reared at $19^{\circ} \mathrm{C}, 31$ at $23^{\circ} \mathrm{C}$, and 38 at $27^{\circ} \mathrm{C}$.

\section{Image analysis of target traits}

The ventral surface of the right forewing and hindwing, and the dorsal surface of the forewing of the eclosed females with undamaged wings were photographed (Leica DC200 digital camera) under a binocular microscope (Leica MZ12) with controlled light and 10× magnification. We included a ruler for conversion from pixels to millimeters and a color reference card (QPcard 201) for background correction. The resulting images were analyzed with a custom macro image processing system using an ImageJ-based open-source Fiji software package [48]. For each trait, areas were calculated by a threshold method in which the image was first converted to black and white and values of intensity under or above user- established threshold values were chosen. The measurements of the white central areas of the smaller more anterior eyespots on the forewing (dorsal and ventral, traits 1 and 3, respectively) and hindwing (trait 5) were excluded because of high measurement error. In total, we measured 19 traits characterizing the area of wings and of various color pattern components (Figure 2). We also counted the number of white eyespot centers on the dorsal surface of the hindwing of the non-injected butterflies [34]. Note that the number of females obtained for each treatment is not necessarily equal to the number of measurements available for the 19 traits. This is because not all traits could be measured in all females (for example, in cases of some damaged wings). Final sample sizes for all traits in all experimental groups are given in Additional file 1 for the non-injected individuals and Additional file 2 for early and late injections, respectively.

\section{Hormone injections}

For each temperature, we had two injection treatments: 'hormone' for injection of a solution of 20E, the biologically-active form of ecdysone [49] and 'control' for injection of the same volume of solvent only. Because the duration of pupal stage varies with temperature, as does the dynamics of ecdysone titers [21], we used \% of the duration of the pupal stage when choosing the injection time points. Injections were done on pupae at two stages corresponding to different phases of the natural ecdysone dynamics [21]: 'early' (at 3\% of the total pupal development time) before ecdysone levels start to increase and 'late' (at $16 \%$ of the total pupal development time) corresponding to the ascending phase of the ecdysone levels (Figure 1A). Pupae were injected $(10 \mu \mathrm{L}$ Hamilton syringe with a $0.3 \mathrm{~mm}$ gauge needle) on the left side in the region of the fifth abdominal segment with $3 \mu \mathrm{L}$ of $0.25 \mu \mathrm{g}$ 20E (Sigma-Aldrich: St Louis, MO, USA hormone stock solution $1 \mathrm{mg} / \mathrm{ml}$ in $100 \%$ ethanol) in insect Ringer's buffer (Merck: Darmstadt, Germany) with vital red artificial coloring (Fluka (Sigma-Aldrich group): Buchs, Switzerland). This hormone concentration was chosen to obtain an optimal balance between hormonal effects and pupal survival (compare with [23]). After injection, pupae were placed back at their respective rearing temperature until emergence, and adults were frozen $\left(-20^{\circ} \mathrm{C}\right)$ until wing analysis. The number of females phenotyped for early injections of control:hormone were $32: 19$ for $19^{\circ} \mathrm{C}, 29: 8$ for $23^{\circ} \mathrm{C}$ and $35: 7$ for $27^{\circ} \mathrm{C}$. For late injections, these numbers were $32: 32$ for $19^{\circ} \mathrm{C}, 23: 30$ for $23^{\circ} \mathrm{C}$ and $34: 32$ for $27^{\circ} \mathrm{C}$. Because not all traits could be measured for each female, the final number of measurements for each trait can be different and are shown in Additional file 2 for early and late injections. Smaller 
sample sizes for early hormone injections are due to higher mortality associated with that treatment.

\section{Hormone titers}

We injected female pupae reared at $19^{\circ} \mathrm{C}$ and $27^{\circ} \mathrm{C}$ with hormone and control solutions at 3\% of the duration of pupal stage, and measured internal $20 \mathrm{E}$ at $3.5 \%$ or at $8.5 \%$ of total pupal development time. For that, we extracted $50 \mu \mathrm{l}$ of hemolymph from each of four pupae per treatment and time point, and measured 20E levels using the ACE enzyme immunoassay (Cayman Chemical Co., Ann Arbor, MI, USA) following the manufacturer's instructions. Briefly, samples were extracted from individual pupae by homogenization followed by addition of $200 \mu \mathrm{l}$ of $70 \%$ methanol. The homogenates were dried using a rotary evaporator at room temperature and dissolved in assay buffer. Calibration curves were generated using commercially available 20E (Sigma; $0.5 \mu \mathrm{g} / \mu \mathrm{l}$ in $100 \%$ ethanol). Absorbance for controls, standards, and hemolymph samples was measured by spectrophotometry at a wavelength of $405 \mathrm{~nm}$ (VICTOR Multilabel Plate Reader). Note that this hormone quantification method can detect concentrations down to a minimum concentration of $7.8 \mathrm{pg} / \mu \mathrm{l}$, which is below the detection level of the method used previously to characterize the titer dynamics displayed in Figure 1A [21].

\section{Immunohistochemistry}

Antibody staining of pupal wings was performed as described in [20] using a custom antibody against B. anynana EcR [43] obtained from ProteinTech Group, Chicago, IL, USA (peptide within region common to all isoforms: CWDVADVNSAQPPPVFDHASDL) at a final dilution of 1:50 (after testing a range of concentrations). The antibody was tested together with other antibodies to assess: 1) specificity by comparing its localization with the Manduca anti-EcR (we observed similar patterns but with less background for the $B$. anynana-specific antibody); 2) detection of the active form of EcR by comparing its localization with that of the known downstream EcR target Broad; 3) association with the eyespot field and intra-cellular localization by comparing with the localization of 4',6diamidino-2-phenylindole (DAPI). We also detected EcR in younger 'clearer' tissues (larval wings) in order to confirm the intra-cellular localization of this antibody. We performed stainings of wings dissected from multiple pupae and covering $6 \%$ to $30 \%$ of pupal duration for each of the two extreme rearing temperatures $27^{\circ} \mathrm{C}$ and $19^{\circ} \mathrm{C}$. The primary anti-EcR antibody was detected with Alexa Fluor 594 anti-rabbit (Molecular Probes, Invitrogen AG, Basel, Switzerland) and images were collected on a Leica DMIRE2, Leica SP5 confocal laser scanning and Nikon Eclipse TE2000-S Screening microscopes.

\section{Statistical analysis of effects of developmental temperature on wing traits}

All data analyses were done using the $R$ statistics package [50] and Mathematica software package [51]. We tested for the effect of temperature on wing traits of noninjected individuals (Figure 3) using ANOVA with temperature as a factor (three levels: $19^{\circ} \mathrm{C}, 23^{\circ} \mathrm{C}, 27^{\circ} \mathrm{C}$ ) and for wing pattern traits 1 to 8 , using the respective wing area as covariate with the model trait $\sim$ wing area + temperature. Trait areas were used untransformed or $\log 10$ transformed to meet the Shapiro-Wilk normality test $(P \geq 0.05)$. When temperature was found to have a significant effect on trait values $(P<0.01)$, we did post-hoc comparisons between pairs of temperatures using lsmeans [see Additional file 1]. To test for the effect of temperature on the number of white pupils on the dorsal surface of the hindwing we used an ANOVA with a Chi-square test and a quasi-Poisson distribution. We tested the model pupil $n r \sim$ temperature, using temperature as a factor (three levels: $19^{\circ} \mathrm{C}, 23^{\circ} \mathrm{C}, 27^{\circ} \mathrm{C}$ ).

\section{Statistical analysis of differences in hormone titers}

We tested for the effect of temperature and injection treatment on the levels of $20 \mathrm{E}$ at two developmental time points (Figure 1B) using the model 20E time point + temperature *injection. We first confirmed that the residuals showed no significant departure from normality (Shapiro-Wilk test) or from homogeneity of variances (Fligner-Killeen test). We then used ANOVA to test for the effect on levels of $20 \mathrm{E}$ of time point (factor with two levels: $3.5 \%$ and $8.5 \%$ ), temperature (factor with levels $19^{\circ} \mathrm{C}$ and $27^{\circ} \mathrm{C}$ ), injection (factor with two levels: hormone and control) and the interaction temperature*injection. Because there was no significant effect of time point, we did pairwise comparisons between temperature and injection groups using Tukey's honest significance tests [see Additional file 4].

\section{Statistical analyses of the effects of hormone manipulations on wing traits}

We tested for the effect of hormone injections, done at different temperatures and at different developmental time points, on wing traits (Figure 4) using core and confirmatory tests in a series of steps. Details of the analyses are shown in Additional file 2. To facilitate between-trait comparisons, we rescaled raw trait measurements to an identical (0 to 1$)$ range. This was done for each of 114 groups (3 temperatures $\times 2$ injection treatments $\times 2$ time points $\times 19$ traits) by setting the minimum trait value to 0 and the maximum value to 1 and rescaling intermediate values proportionally. We then checked the normal distribution of the rescaled trait values in each group (JarqueBera test, alpha $=0.01$ ). For normally distributed values, we used a two-tailed $\mathrm{T}$ test to compare control and 
hormone treatment means for each trait, temperature and time point. For the one non-normally distributed group values (hindwing area, trait 10, after early injection at $27^{\circ} \mathrm{C}$ ), we used a two-tailed Mann-Whitney $\mathrm{U}$ test to compare control and hormone medians. We used the false discovery rate procedure [52] with alpha $=0.05$ to determine the contextual significance of each of the $57 P$-values obtained per injection time point.

To take into account differences across treatments in sample size and, particularly, the reduced sample sizes in the early hormone injection groups [23], we carried out an extra validation statistical analysis. We combined two types of resampling techniques [53]: (1) bootstrap (a good method to estimate population parameter differences from small samples); and (2) permutation tests to determine the significance ( $P$-values) of the parameter differences (or displacements) obtained via the bootstrap distributions. We performed a bootstrap-based estimation of the displacement of mean/median for each group by resampling 1,000 times from the original distributions of trait values (keeping sample size with replacement). Because the bootstrap distributions did not depart significantly from normality (Jarque-Bera test, alpha = 0.01 ), we used the mean of that distribution as the estimator of mean displacement (difference) between control and hormone-injected groups. We then used permutation tests to compare differences between control and hormone injections (for each trait, temperature and time point) assessed from the original dataset with those from the resampled dataset. For each of the 57 pairs (19 traits $\times 3$ temperatures $\times 2$ time points) of control and hormone groups, we computed the difference between their original means, and then estimated mean difference 1,000 times from resampled data as follows (note that only means were used on the basis that no bootstrap distribution for the previous goal departs significantly from normality): 1) we merged the two distributions (control with hormone values) into a single distribution; 2) 1,000 times, we divided the values in this distribution into two groups of the same sizes as the original control and hormone groups; 3) we calculated the mean difference between these groups; 4) we thus produced a list of 1,000 mean differences (in absolute value); and 5) we calculated a $P$-value for our original comparison of control versus hormone means as the proportion of those 1,000 values that is different from the original mean difference divided by 1,000 (two-tailed test). The $P$-values obtained were also subjected to the false discovery rate procedure [52] with alpha $=0.05$ to determine the contextual significance of each of the $57 P$-values obtained per injection time point. We compared both sets of results obtained from the core test (k-sample t-test or Mann-Whitney as appropriate) and from permutation tests and found them to be not in conflict [see Additional file 2].

\section{Additional files}

Additional file 1: Results S1. Summary of ANOVA results to test the effect of temperature on wing traits of non-injected individuals (compare with Figure 3).

Additional file 2: Results S2. Summary of statistical analyses for wing trait values upon early and late control and hormone injections. This file supports results in Figure 4 and contains Tables S1 (for early injections) and S2 (for late injections) displaying sample sizes, mean and standard error of the re-scaled trait values, difference between hormone and control values (before and after bootstrap), as well as the $p$-values for the statistical significance of those differences.

Additional file 3: Figure S1. Hormone injection phenocopies effects of higher developmental temperature. This figure shows the extent to which hormone manipulations at lower temperatures increase trait areas to levels characteristic of higher temperatures.

Additional file 4: Results S3. Summary of ANOVA results to test the effect of temperature and injection treatment on the levels of $20 \mathrm{E}$ at two developmental time points (compare with Figure 1B).

Additional file 5: Figure S2. Patterns of coordinated response to external and internal signals. This figure illustrates which traits responded in concert and in contrast to either the temperature treatment (compare with Figure 3) or the hormone manipulations (compare with Figure 4) and shows in detail the findings summarized in Figure 5.

Additional file 6: Results S4. Principal components analysis (PCA) for variation in eyespot traits, separately for non-injected individuals (compare with Figure 3) and for hormone manipulations (compare with Figure 4). This file contains the material and methods, figures S3 and S4, as well as results and discussion of the PCA.

\section{Competing interests}

The authors declare that they have no competing interests.

\section{Authors' contributions}

ARAM, VO, PMB, BZ and PB designed the study; ARAM, VO and EL collected the data; ARAM, MMP and PB analyzed the data; ARAM, MMP and PB interpreted the results; PB and ARAM wrote the manuscript. All authors read and approved the final manuscript.

\section{Acknowledgements}

We thank E. van Bergen, T. Piessens, M. van Eijk and K. Koops for help processing butterflies; P. Almada for help with image analysis; S. Dickinson, D. Duneau and N. Martins for guidance on statistical analyses; T. Koyama and C. K. Mirth for advice about measuring $20 \mathrm{H}$ titers; L. Riddiford for providing antibodies against Manduca sexta EcR and its downstream targets; K. Tanaka for help with immunohistochemistry; D. Duneau for comments on the manuscript. We also thank the helpful suggestions of two anonymous reviewers. The authors wish to acknowledge funding from the Portuguese Foundation for Science and Technology FCT (fellowships SFRH/BD/45486/ 2008 to ARA Mateus and PTDC/EIA-CCO/114108/2009 to M. Marques-Pita, and research grant PTDC/BIA-BDE/100243/2008 to P. Beldade), and from the EU FP6 (Network of Excellence LifeSpan FP6 036894) and FP7 (IDEAL FP7/2007-2011/259679) to B.J. Zwaan and V. Oostra and the Netherlands Organization for Scientific Research NWO.

\section{Author details}

${ }^{1}$ Instituto Gulbenkian de Ciência, Rua da Quinta Grande 6, P-2780 Oeiras, Portugal. ${ }^{2}$ Institute of Biology, Leiden University, Sylviusweg 72, 2333, BE Leiden, The Netherlands. ${ }^{3}$ School of Informatics and Computing, Indiana University, 919 East Tenth Street, Bloomington, IN 47408, USA. ${ }^{4}$ Department of Zoology, Cambridge University, Downing Street, Cambridge CB2 3EJ, UK. ${ }^{5}$ Laboratory of Genetics, Wageningen University, Droevendaalsesteeg 1, 6708 PB Wageningen, The Netherlands.

Received: 5 September 2014 Accepted: 4 November 2014 Published: 21 November 2014 


\section{References}

1. Beldade P, Mateus AR, Keller RA: Evolution and molecular mechanisms of adaptive developmental plasticity. Mol Ecol 2011, 20:1347-1363.

2. Miura T: Developmental regulation of caste-specific characters in social-insect polyphenism. Evol Dev 2005, 7:122-129.

3. Schwander T, Lo N, Beekman M, Oldroyd BP, Keller L: Nature versus nurture in social insect caste differentiation. Trends Ecol Evol 2010, 25:275-282.

4. Keller RA, Peeters C, Beldade P: Evolution of thorax architecture in ant castes highlights trade-off between flight and ground behaviors. elife 2014, 3:e01539.

5. Simpson SJ, Sword GA, Lo N: Polyphenism in insects. Curr Biol 2011, 21:738-749.

6. Brakefield PM, French V: Butterfly wings: the evolution of development of color patterns. Bioessays 1999, 21:391-401.

7. Nijhout HF: Development and evolution of adaptive polyphenisms. Evol Dev 2003, 5:9-18.

8. Guthrie HA, Brown ML: Effect of severe undernutrition in early life on growth, brain size and composition in adult rats. J Nur 1968, 94:419-426.

9. David JR, Gilbert P, Pla E, Petavy P, Karan D, Moreteau B: Cold stress tolerance in Drosophila: analysis of chill coma recovery in D. melanogaster. J Therm Biol 1998, 23:291-299.

10. Shingleton AW, Estep CM, Driscoll MV, Dworkin I: Many ways to be small: different environmental regulators of size generate distinct scaling relationships in Drosophila melanogaster. Proc R Soc Lond B 2009, 276:2625-2633.

11. Cheverud JM: Developmental integration and the evolution of pleiotropy. Am Zool 1996, 36:44-50.

12. Wagner GP: Homologues, natural kinds and the evolution of modularity. Am Zool 1996, 36:36-43.

13. Ketterson ED, Atwell JW, McGlothlin JW: Phenotypic integration and independence: hormones, performance, and response to environmental change. Integr Comp Biol 2009, 49:365-379.

14. Tang HY, Smith-Caldas MH, Driscoll MV, Salhadar S, Shingleton AW: FOXO regulates organ-specific phenotypic plasticity in Drosophila. PLoS Genet 2011, 7:e1002373.

15. Koyama T, Mendes CC, Mirth CK: Mechanisms regulating nutritiondependent developmental plasticity through organ-specific effects in insects. Front Physio/ 2013, 4:263

16. Brakefield PM, Gates J, Keys D, Kesbeke F, Wijngaarden P, Monteiro A, French V, Carroll S: Development, plasticity and evolution of butterfly eyespot patterns. Nature 1996, 384:236-242.

17. Schlichting CD, Pigliucci M: Phenotypic Evolution: A Reaction Norm Perspective. MA: Sinauer Associates, Sunderland, Massachusetts, USA; 1998

18. Gilbert SF, Epel D: Ecological Developmental Biology: Integrating Epigenetics, Medicine, and Evolution. MA: Sinauer Associates, Inc:; 2009

19. Beldade P, Brakefield PM: The genetics and evo-devo of butterfly wing patterns. Nat Rev Genet 2002, 3:442-452

20. Brakefield PM, Beldade P, Zwaan BJ: The African butterfly Bicyclus anynana: a model for evolutionary genetics and evolutionary developmental biology. In Emerging Model Organisms: A Laboratory Manual. Edited by Behringer RR, Johnson AD, Krumlauf RE. New York: Cold Spring Harbor Laboratory Press; 2009:291-329.

21. Oostra V, de Jong MA, Invergo B, Kesbeke F, Wende F, Brakefield PM, Zwaan $B J$ : Translating environmental gradients into discontinuous reaction norms via hormone signaling in a polyphenic butterfly. Proc $R$ Soc Lond $B$ 2011, 278:789-797

22. Brakefield PM, Kesbeke F, Koch PB: The regulation of phenotypic plasticity of eyespots in the butterfly Bicyclus anynana. Amer Nat 1998, 152:853-860.

23. Koch PB, Brakefield PM, Kesbeke F: Ecdysteroids control eyespot size and wing color pattern in the polyphenic butterfly Bicyclus anynana (Lepidoptera: Satyridae). J Insect Physiol 1996, 42:223-230.

24. Zijlstra WG, Steigenga MJ, Koch PB, Zwaan BJ, Brakefield PM: Butterfly selected lines explore the hormonal basis of interactions between life histories and morphology. Amer Nat 2004, 163:76-87.

25. Prudic $\mathrm{KL}$, Jeon $\mathrm{C}$, Cao H, Monteiro A: Developmental plasticity in sexual roles of butterfly species drives mutual sexual ornamentation. Science 2011, 331:73-75.

26. Oliver JM, Robertson KA, Monteiro A: Accommodating natural and sexual selection in butterfly wing pattern evolution. Proc $R$ Soc Lond B 2009, 276:2369-2375.

27. Beldade P, Brakefield PM: Concerted evolution and developmental integration in modular butterfly wing patterns. Evol Dev 2003, 5:169-179.

28. Beldade P, Koops K, Brakefield PM: Developmental constraints versus flexibility in morphological evolution. Nature 2002, 416:844-847.

29. Beldade P, Koops K, Brakefield PM: Modularity, individuality, and evo-devo in butterfly wings. Proc Natl Acad Sci U S A 2002, 99:14262-14267.
30. Allen CE, Beldade P, Zwaan BJ, Brakefield PM: Differences in the selection response of serially repeated color pattern characters: standing variation, development, and evolution. BMC Evol Biol 2008, 8:94

31. Saenko SV, Brakefield PM, Beldade P: Single locus affects embryonic segment polarity and multiple aspects of an adult evolutionary novelty. BMC Biol 2010, 8:111.

32. Brakefield PM, Frankino WA: Polyphenisms in Lepidoptera: multidisciplinary approaches to studies of evolution. In Phenotypic Plasticity in Insects. Edited by Ananthakrishnan TN, Whitman DW. Plymouth UK: Science Publishers, Inc; 2007:121-151.

33. Breuker CJ, Brakefield PM: Female choice depends on size but not symmetry of dorsal eyespots in the butterfly Bicyclus anynana. Proc R Soc Lond B 2002, 269:1233-1239.

34. Westerman EL, Chirathivat N, Schyling E, Monteiro A: Mate preference for a phenotypically plastic trait is learned, and may facilitate preferencephenotype matching. Evolution 2014, 68:1661-1670.

35. Saenko SV, Marialva MS, Beldade P: Involvement of the conserved Hox gene Antennapedia in the development and evolution of a novel trait. EvoDevo 2011, 2:9.

36. Nijhout HF, Cinderella M, Grunert LW: The development of wing shape in Lepidoptera: mitotic density, not orientation, is the primary determinant of shape. Evol Dev 2014, 16:68-77.

37. Koch PB, Lorenz U, Brakefield PM, ffrench-Constant RH: Butterfly wing pattern mutants: developmental heterochrony and co-ordinately regulated phenotypes. Dev Genes Evol 2000, 210:536-544

38. ffrench-Constant $\mathrm{RH}$ : Butterfly wing colors are driven by the evolution of developmental heterochrony. Butterfly wing colors and patterning by numbers. Heredity 2012, 108:592-593.

39. Oostra V, Mateus AR, van der Burg KR, Piessens T, van Eijk M, Brakefield PM, Beldade P, Zwaan BJ: Ecdysteroid hormones link the juvenile environment to alternative adult life histories in a seasonal insect. Amer Nat 2014, 184:79-92.

40. Brakefield PM, French V: Eyespot development on butterfly wings: the focal signal. Dev Biol 1995, 168:112-123.

41. Koch PB, Merk R, Reinhardt R, Weber P: Localization of ecdysone recepto protein during color pattern formation in wings of the butterfly Precis coenia (Lepidoptera: Nymphalidae) and co-expression with Distal-less protein. Dev Genes Evol 2003, 212:571-584.

42. Beldade P, Brakefield PM, Long AD: Contribution of Distal-less to quantitative variation in butterfly eyespots. Nature 2002, 415:315-318.

43. Conceição IC, Long AD, Gruber JD, Beldade P: Genomic sequence around butterfly wing development genes: annotation and comparative analysis. PLoS One 2011, 6:e23778.

44. Oliver JC, Ramos D, Prudic KL, Monteiro A: Temporal gene expression variation associated with eyespot size plasticity in Bicyclus anynana. PLoS One 2013, 8:e65830.

45. Maynard Smith J, Burian R, Kauffman S, Alberch J, Campbell B, Goodwin R, Lande R, Raup D, Wolpert L: Developmental constraints and evolution. Quart Rev Biol 1985, 60:265-287.

46. Hau M: Regulation of male traits by testosterone: implications for the evolution of vertebrate life histories. Bioessays 2007, 29:133-144.

47. West-Eberhard MJ: Developmental plasticity and the origin of species differences. Proc Natl Acad Sci U S A 2005, 102:6543-6549.

48. Schindelin J, Arganda-Carreras I, Frise E, Kaynig V, Longair M, Preibisch S, Rueden C, Saalfeld S, Schmid B, Tinevez J, Hartenstein V, Eliceiri K, Tomancak P, Cardona A: Fiji - an open source platform for biological image analysis. Nat Methods 2012, 9:676-682.

49. Richards GP: Insect hormones in development. Biol Rev 1981, 56:501-549.

50. R-Core-Team: $R$ - A Language and Environment for Statistical Computing. 2012. http://www.R-project.org/

51. Wolfram S: Mathematica 3.0 User's Guide. New York: Cambridge University Press; 1996.

52. Benjamini Y, Hochberg Y: Controlling the False Discovery Rate: a practical and powerful approach to multiple testing. J R Statist SoC B 1995, 57:289-300.

53. Efron B, Tibshirani RJ: An Introduction to the Bootstrap. New York: Chapman \& Hall; 1993

doi:10.1186/s12915-014-0097-x

Cite this article as: Mateus et al:: Adaptive developmental plasticity:

Compartmentalized responses to environmental cues and to corresponding internal signals provide phenotypic flexibility. BMC Biology 2014 12:97. 